\section{Ahd El-Basit W., Ahd El-Maksood A. M.}

\title{
STUDY OF GAMMA-RAY EFFECTS ON RELAXATION OSCILLATOR BASED ON UNIJUNCTION TRANSISTOR
}

The object of this research is all the semiconductor unijunction transistor device (UJT) parameters that affect its operation as $1.67 \mathrm{kHz}$ saw-tooth relaxation oscillator circuit under the influence of gamma-irradiation dose. Relaxation oscillators are widely used in function generators, electronic beepers, inverters, blinkers, and voltagecontrolled oscillators. The properties of UJTs are like those of other semiconductor devices are greatly affect by irradiation. Its electrical characteristics and the output voltage waveforms of the relaxation oscillator circuit were investigated and plotted as a function of different gamma-dose levels. The type of semiconductor, the design of the device and the type of radiation are affected the magnitude of this change. Where, it is shown that increasing gamma dose up to $3.0 \mathrm{MGy}$ leads, the peak voltage $\left(V_{P}\right)$ to be decreased from 3.71 Volts down to 2.9 Volts, while the valley voltage $\left(V_{V}\right)$ to be increases from 1.54 Volts up to 1.89 Volts, leading to a pronounced narrowing on the negative resistance region. As a result, both the output signal voltage amplitude and the frequency of the relaxation oscillator circuit were shown to be functions of the gamma-irradiation dose. Where, the initial values of the output signal voltage amplitude were reported to be 2.54 Volts decreased to the value of 2.13 Volts, while its frequency was $1.67 \mathrm{kHz}$ increased up to $1.85 \mathrm{kHz}$, respectively, due to gamma-exposure levels up to $3.0 \mathrm{MGy}$ using the GammaCell-220 (National Center for Radiation Research and Technology of Egypt).

Keywords: semiconductor unijunction transistor device, relaxation oscillator, gamma-irradiation dose, gammaexposure levels.

Received date: 08.04.2020

Accepted date: 25.05 .2020

Published date: 31.08 .2020
Copyright (C) 2020, Abd El-Basit W., Abd El-Maksood A.M. This is an open access article under the CC BY license (http://creativecommons.org/licenses/by/4.0)

\section{Introduction}

All devices utilized by humaneness are constantly exposed to different kinds of radiation originating from natural sources, however additionally from artificial manmade sources. Increasing the amount of integration of electronic components and miniaturization trends might have negative influence onto the components sensitivity to radiation. Radiation defects can also occur in a process of very large-scale integrated circuits fabrication, since the process often includes bombardment with high-energy ions and photons [1]. An electronic device which works in a radiation environment can present degradation due to the damage occurred in the semiconductor lattice [2].

The object of this research is all the semiconductor unijunction transistor device (UJT) parameters that affect its operation as $1.67 \mathrm{kHz}$ saw-tooth relaxation oscillator circuit under the influence of gamma-irradiation dose.

The aim of this paper is to investigate the influence of gamma-irradiation up to 3.0 MGy on the operation of a $1.67 \mathrm{kHz}$ saw-tooth relaxation oscillator circuit based on the UJT. In this concern, its emitter current-voltage $\left(I_{E}-V_{E}\right)$, peak- and valley-voltages $\left(V_{P}, V_{V}\right)$, inter-base resistance $\left(R_{B B}\right)$ and inter-base voltage-current were investigated, as well, the electrical output parameters of the relaxation oscillator circuit.

\section{Methods of research}

2.1. Construction and characteristics of unijunction transistor. The UniJunction transistor (UJT), also called «double-base diode», was discovered by Jerry Suran in 1953 [3].

A unijunction transistor is a three-terminal device exhibiting a negative resistance region under certain conditions [4]. It is made of a lightly doped N-type silicon bar with two ohmic contacts called a base, to which a small amount of $p$-type material is diffused. The base terminals connected to each end are labeled B1 (base 1) and B2 (base 2). The P-material is the emitter and is labeled $E$. A power supply is connected across the base with the negative lead at B1 and the positive lead at B2 (Fig. 1) [5, 6]. The electrical equivalent circuit comprises a potential divider arrangement of two resistors $R_{B 1}$ and $R_{B 2}$ and a PN junction diode. Resistor $R_{B 1}$ represents the resistance of the base bar between B1 terminal and the PN junction and resistor $R_{B 2}$ represents the resistance of the base bar between $\mathrm{B} 2$ terminal and the PN junction. $R_{B 1}$ has been shown as a variable resistance as its value depends on the emitter current flowing through the PN junction when it is forward biased. For $I_{E}=0$, the total resistance of the base bar is termed as $R_{B B}\left(R_{B B}=R_{B 1}+R_{B 2}\right) . R_{B B}$ lies in the range of $4 \mathrm{~K} \Omega$ to $10 \mathrm{~K} \Omega$. Another parameter defined for 
a UJT is the intrinsic stand-off ratio denoted by $\eta$, where it is given by equation [7]:

$$
\eta=R_{B 1} /\left(R_{B 1}+R_{B 2}\right) \text {. }
$$

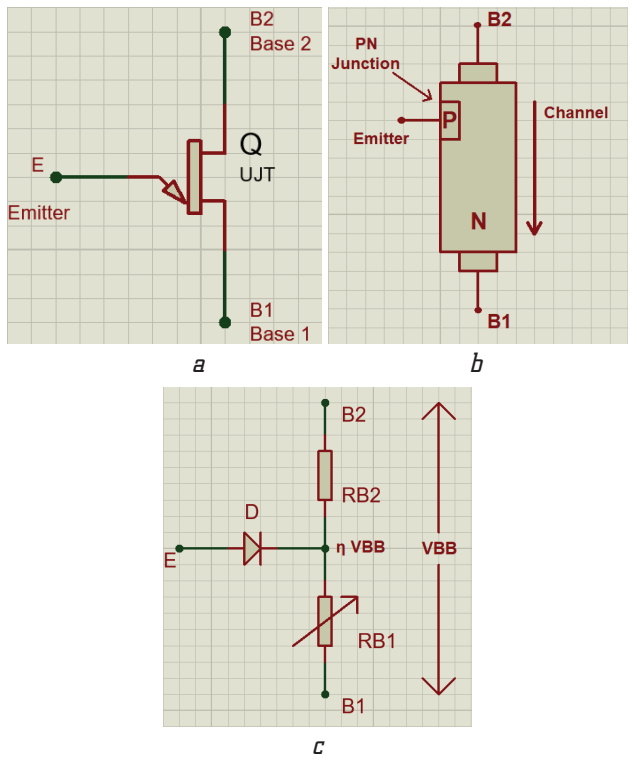

Fig. 1. Unijunction transistor: $a$ - schematic symbol; $b$ - structure diagram; $c$ - equivalent circuit

2.2. Unijunction transistor relaxation oscillator. Oscillator is an essential block in communication and biomedical systems [8]. In this work the design of relaxation oscillator based on UJT is proposed using type 2N4870. Unijunction transistors are widely used in industries as a voltage controlled negative resistance relaxation oscillator [9] because of resistivity of UJT varies owing to carrier injection. Due to this, on the characteristics of unijunction transistor appears a domain with negative resistance, that is, under certain conditions, the voltage across the transistor can be reduced even with an increase in the output current through the load of transistor. Only the turn off transistor or opening the circuit can remove the input voltage [10].

The UJT has been mainly used as active device in relaxation oscillators because its current-voltage static characteristics have a portion in which the resistance is negative at the emitter terminal $(E)$. The static emitter characteristic, i. e. the mathematical function modeling the relationship between the emitter voltage $V_{E}$ and the emitter current $I_{E}$ of a UJT at a given inter base voltage $V_{B}$ is plotted in Fig. 2 [11].

A relaxation oscillator consists of an energy-storing element such as a capacitor and a nonlinear switching device such a Schmitt trigger comparator or negative resistance element connected by a feedback loop. The switching device periodically charges and discharges the energy stored in the capacitor, yielding abrupt changes in the output waveform [12]. The proposed relaxation oscillator circuit shown in Fig. 3, $a$ consists of a UJT and a capacitor $C$ which is charged through $R$ as $V$ is switched on. When the capacitor voltage $V_{C}$ reaches in time $t_{s}$ the value of $V_{P}$, the UJT fires and rapidly discharges $C$ via $B_{1}$ till the voltage falls below the minimum value $V_{V}$. The device then cuts off and $C$ starts to charge again. This cycle is repeated continuously thus generating a saw-tooth waveform across $C$. It should be noted that charging time constant of the capacitor for voltage $V$ is $T=C R$ whereas discharging time constant is $T_{d}=C R_{B 1}$. The time required to charge up to $V_{P}$ (called ramp rise time) is:

$$
t_{s}=T \cdot \log _{e}\left(V-V_{V}\right) /\left(V-V_{P}\right) \text {. }
$$

Similarly, time required by the capacitor to discharge from $V_{P}$ to $V_{V}$ is:

$$
t_{d} \cong T_{d} \cdot \log _{e} \cdot V_{P} / V_{V}
$$

The frequency of oscillation is given by [13]:

$$
f=1 /\left(t_{s}+t_{d}\right)
$$

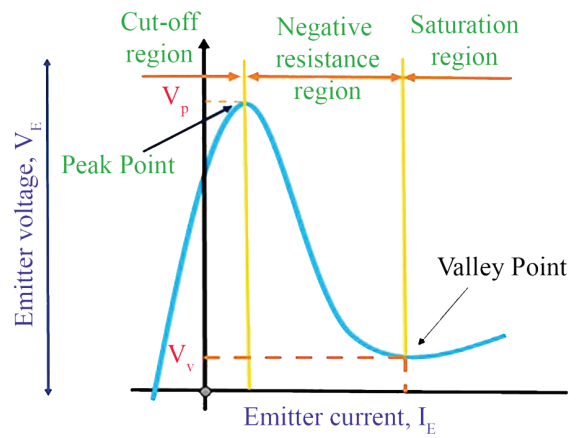

Fig. 2. Static Emitter Characteristic of the unijunction transistor
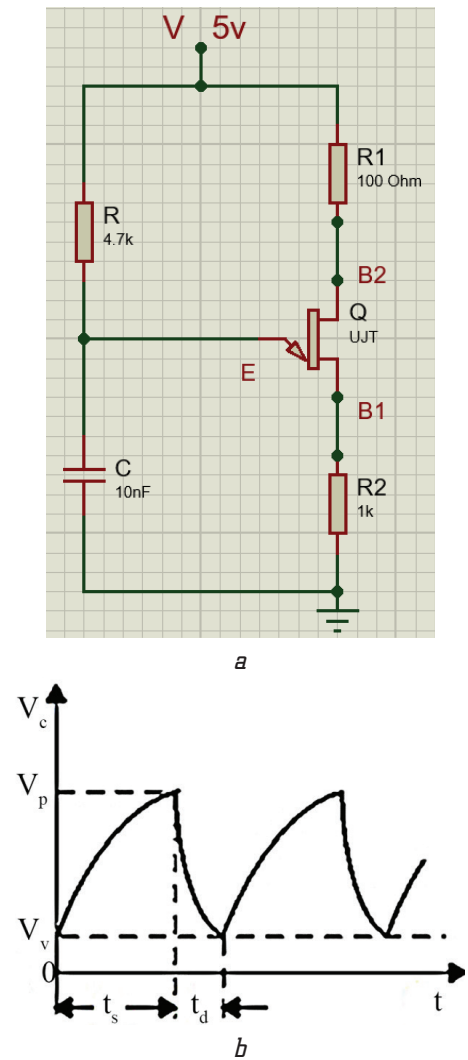

Fig. 3. A schematic representation of: a - unijunction transistor relaxation oscillator circuit; $b$ - output saw-tooth waveform across $C$

Relaxation oscillators are generally used to create a low frequency signals for such applications as blinking lights and electronic beepers and clock signals in some digital circuits. The term relaxation oscillator is also applied to dynamical systems in many diverse areas of science that produce 
a nonlinear oscillation that produces a non-sinusoidal repetitive output signal. And can be analyzed using the same mathematical model as electronic relaxation oscillators [14].

2.3. Experimental procedures. In order to obtain complete data about the effects of gamma-irradiation on Unijunction transistor (UJT) type $2 \mathrm{~N} 4870$, as a relaxation oscillator circuit, its characteristics were plotted at different ascending gamma-doses up to 3.0 MGy using the GammaCell-220 (National Center for Radiation Research and Technology of Egypt) [15]. The electrical parameters based on $I-V$ characteristics are investigated using the circuit shown in Fig. 4.

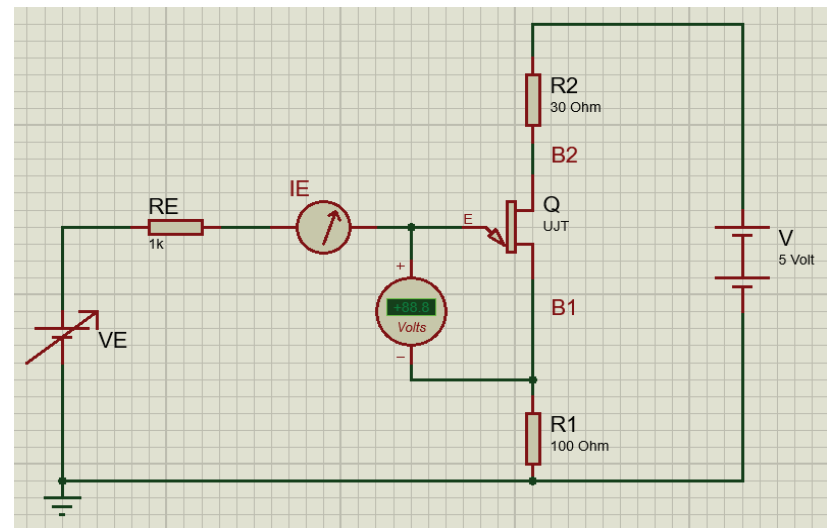

Fig. 4. Characterization circuit of unijunction transistors

In addition, a schematic representation of a driven UJT relaxation oscillator circuit is designed, constructed and implemented to produce a saw-tooth wave at a frequency of $1.67 \mathrm{kHz}$ (Fig. 3), where waveforms were traced using a highresolution oscilloscope «Tektronix Model TDS2024C (USA).

\section{Research results and discussion}

3.1. Radiation effect on the electrical parameters of UJT devices. The properties of UJTs are like those of other semiconductor devices which are greatly influenced by irradiation. So, it is expected that both branches of the $\left(I_{E}-V_{E}\right)$ characteristic curves and electrical parameters of the devices change due to gamma-exposure dose levels. The magnitude of this change depends on the type of semiconductor, on the design of the device and the type of radiation. This part of the work is devoted to a discussion of permanent radiation effects on the tested UJT device type $2 \mathrm{~N} 4870$ [16-18]. The negative resistance characteristic of the unijunction transistor depends on the conductivity modulation of a moderately high resistivity silicon bar by means of injected minority carriers from the rectifying emitter contact. It is, therefore, highly sensitive to radiation induced changes in minority carrier lifetime and resistivity. Fig. 5 shows a typical unijunction characteristic at constant value of $V_{B 2 B 1}$ equal 5 Volts. Gamma radiation will produce some displacement damage. However, gamma radiation can cause considerable ionization. The effects of ionization on unijunction devices would result primarily in an increase of surface leakage currents with some changes in $V_{V}$ and $V_{p}$ at high dose levels. It is clearly shown that, increasing gamma dose levels from 0.0 up to 3.0 MGy cause the peak voltage point $\left(V_{P}\right)$ to decrease from 3.71 Volts down to 2.9 Volts (Fig. 6).
Gamma radiation data indicate that additional decreases in $V_{P}$ may be incurred due to ionization effects. While the valley voltage $\left(V_{V}\right)$ tends to increase from 1.54 Volts up to 1.89 Volts (Fig. 6), which leads to narrowing the negative resistance region. The peak voltage $V_{P}$ is the most sensitive parameter as opposed to the valley voltage $V_{V}$ for displacement damage [19].

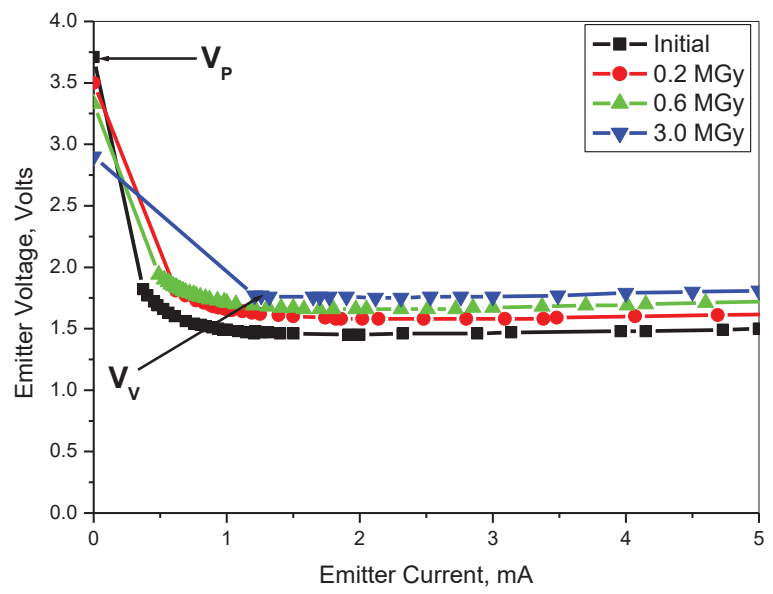

Fig. 5. Current-voltage characteristics of the unijunction transistor type 2N4870 under the influence of gamma exposure

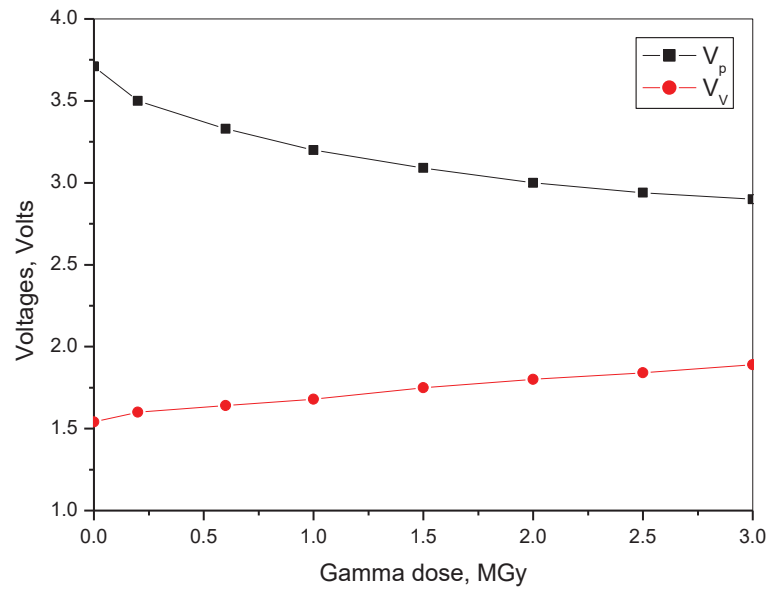

Fig. 6. Dependency of peak $\left(V_{P}\right)$ and valley $\left(V_{V}\right)$ voltages on gamma radiation

The effect of gamma dose on the inter-base characteristics which is considered to be the most important characteristics of the device is shown in Fig. 7. It is noticed that, the base current $\left(I_{B}\right)$ increases linearly as a function of increasing both; inter-base voltage (Fig. 7, $a$ ) and gamma dose levels (Fig. 7, $b$ ). Where at a certain inter-base voltage value of 5.0 Volts, as an example, it is clearly shown that, $I_{B}$ increase from $6.5 \mathrm{~mA}$ up to $8.5 \mathrm{~mA}$.

The change of inter-base resistance in $2 \mathrm{~N} 4870 \mathrm{Si}-\mathrm{UJT}$ under irradiation of ${ }^{60} \mathrm{Co} \gamma$-ray is provided. Through measurement, it was shown that the inter-base resistance decreased exponentially from $8.33 \mathrm{k} \Omega$ down to $6.5 \mathrm{k} \Omega$ and then constant as shown in Fig. 8. The obtained results were shown to be in excellent agreement with the previously published work on the field. This proves that the displacement effect is the main effect of inter-base resistance in Si-UJT irradiated by ${ }^{60} \mathrm{Co} \gamma$-ray, but it lags behind the ionization effect from microanalysis of the interaction 
between $\gamma$-ray and Si material. This is very important for the radiation hardened research [20].
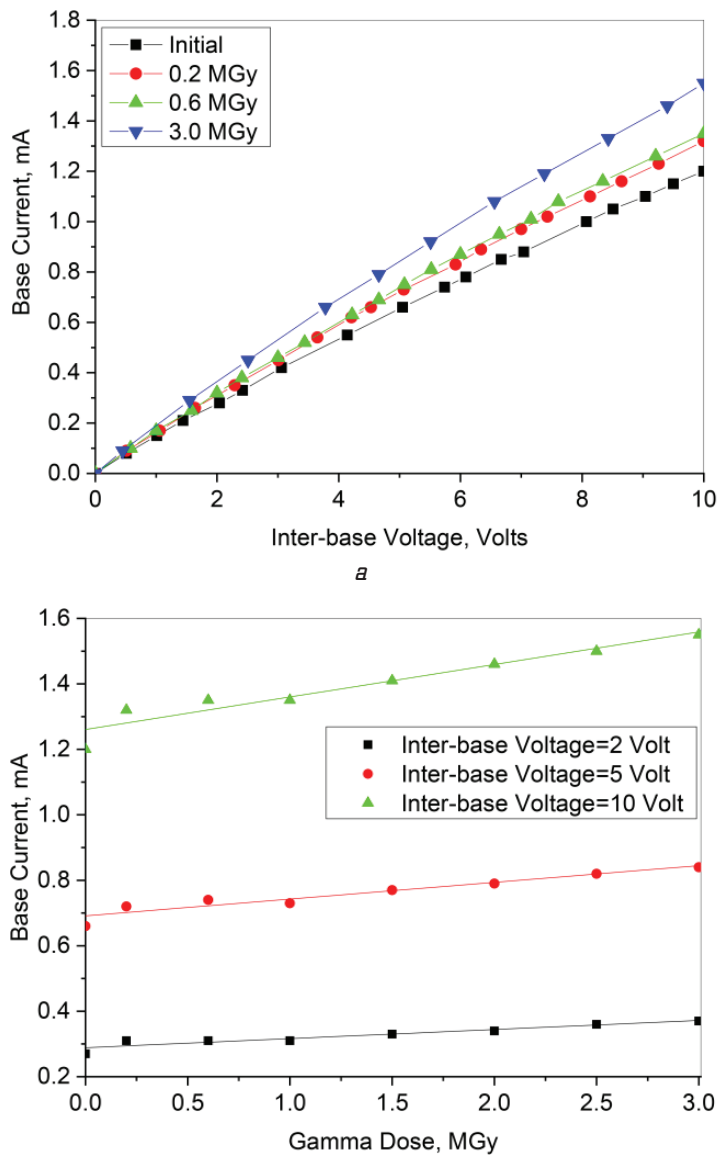

$b$

Fig. 7. Base current of the unijunction transistor type 2N4870 at $I_{E}=0$ as a function of: $a$ - inter-base voltage; $b$ - gamma dose

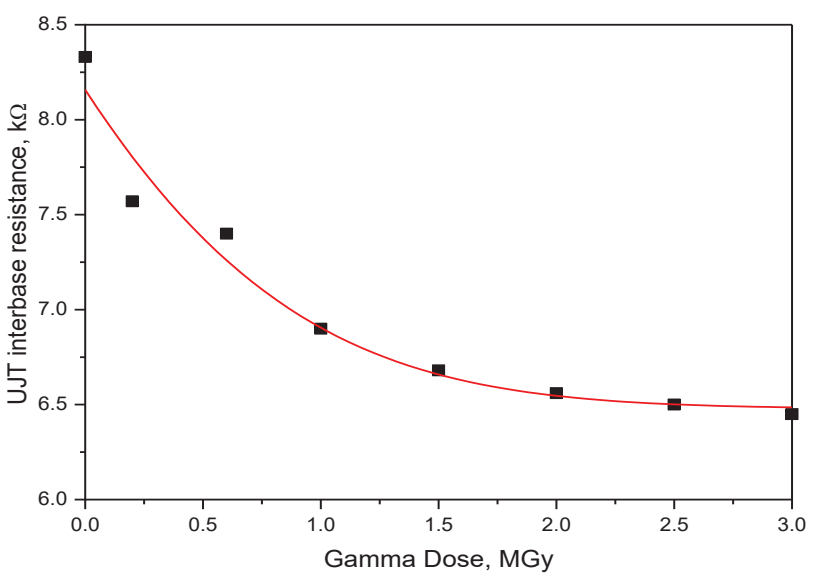

Fig. 8. Inter-base resistance variations as a function of gamma dose

3.2. Radiation effect on the relaxation oscillator circuit. The case of studying the characteristics of the proposed relaxation oscillator circuit under the influence of the gammadose levels is investigated and plotted in Figs. 9, 10.

Fig. 9 shows the output voltage waveforms of UJT relaxation oscillator circuit at the different gamma exposure. It is clearly shown that as the gamma-dose level increases, the following transient times of the pulse interval $(t)$ and the capacitor charge $\left(t_{s}\right)$ as well discharge $\left(t_{d}\right)$ of the output waveforms for the unijunction transistor relaxation oscillator decrease as shown in Fig. 9, 10. Where, values of 540, 534 and $6 \mu \mathrm{sec}$ were recorded, at a gamma dose of $3.0 \mathrm{MGy}$, for $t, t_{s}$ and $t_{d}$, respectively, although their initial values were 600,590 and $10 \mu \mathrm{sec}$, respectively. Also, it is clearly shown that, the dependence of the signal amplitude values decreases from $2.54 \mathrm{~V}$ down to $2.13 \mathrm{~V}$ - by the ratio of $16 \%$ - through the range from initial up to $3.0 \mathrm{MGy}$. While the frequency of the obtained output voltage increases from $1.67 \mathrm{kHz}$ to $1.85 \mathrm{kHz}$ - by the ratio of $11 \%$ - with increasing gamma dose levels.

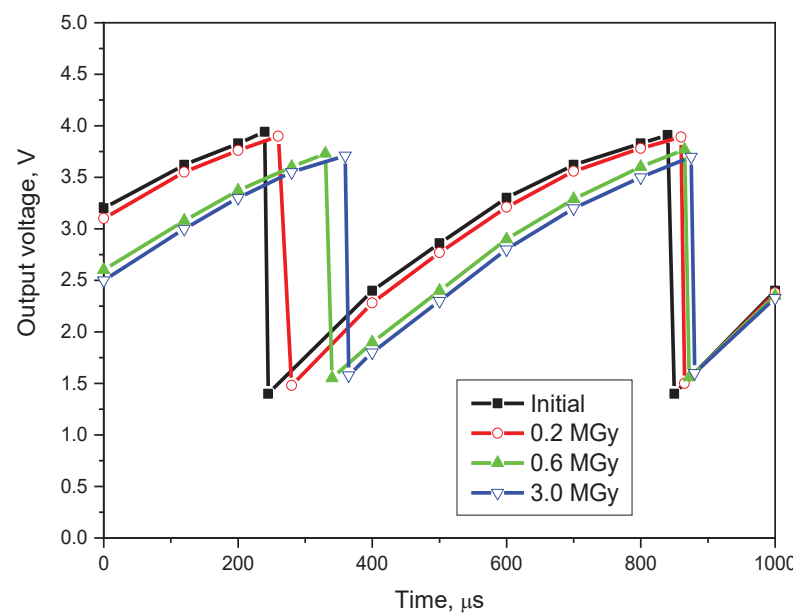

Fig. 9. Dutput voltage waveforms of unijunction transistor relaxation oscillator circuit at the different gamma exposure

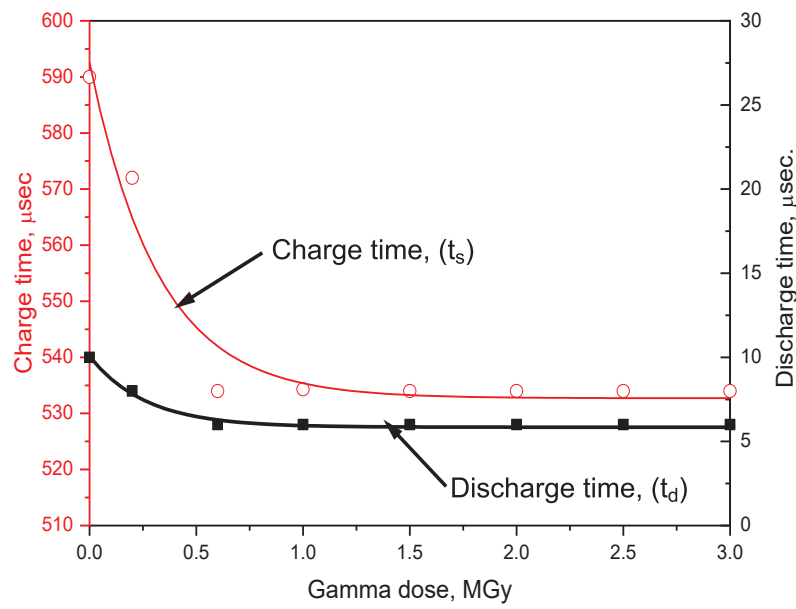

Fig. 10. Gamma radiation effect on the transient times of unijunction transistor relaxation oscillator

\section{Conclusions}

In this work, the effect of gamma-radiation exposure on the characteristics and the performance of $1.67 \mathrm{kHz}$ a saw-tooth relaxation oscillator circuit including a unijunction transistor which, in turn, are used for timing purposes, were investigated experimentally. It is concluded that, the electrical properties of UJTs are greatly influenced by irradiation, like those of other semiconductor devices. The magnitude of this change depends on the type of semiconductor, on the design of the device and the type of radiation which are mainly attributed to the permanent radiation effect on the UJTs. Moreover, the proposed oscillator circuit output waveform shows both amplitude and frequency variations 
as functions of change gamma dose exposure levels. Also, transient times of the pulse interval $(t)$ and the capacitor charge $\left(t_{s}\right)$ as well discharge $\left(t_{d}\right)$ of the output waveforms for the unijunction transistor relaxation oscillator decrease as the gamma dose levels increase.

\section{References}

1. Fetahović, I., Pejović, M., Vujisić, M. (2013). Radiation Damage in Electronic Memory Devices. International Journal of Photoenergy, 2013, 1-5. doi: http://doi.org/10.1155/2013/170269

2. Holmes-Siedle, A., Adams, L. (2002). Handbook of Radiation Effects. Oxford: Oxford University Press, 642.

3. Managt Sci Tech, I. (2014). Study of the Emitter Characteristics $\left(I_{E}-V_{E}\right)$ of UJT at Different Temperatures. International Research Journal of Management Science \& Technology, 5 (6), 23-26.

4. Waller, W. F. (Ed.) (1972). Rectifier Circuits. Macmillan Press Limited. doi: http://doi.org/10.1007/978-1-349-01200-8

5. Bartelt, T. L. M. (2011). Industrial Automated Systems: Instrumentation and Motion Control. Delmar: Cengage Learning.

6. Salivahanan, S., Suresh Kumar, N., Vallavara, A. (2008). Electronic Devices and Circuits. Tata McGraw-Hill Education.

7. Abrar, M. M. (2018). Experimental investigation of synchronized UJT trigger circuitusing UJT 2N2646. International Journal of Advanced Technology and Engineering Exploration, 5 (47), 369-375. doi: http://doi.org/10.19101/ijatee.2018.546023

8. El-Naggar, A. M., Fouda, M. E., Madian, A. H., Radwan, A. G. (2016). Reactance-less RM relaxation oscillator using exponential memristor model. 2016 28th International Conference on Microelectronics (ICM), 361-364. doi: http://doi.org/10.1109/ icm.2016.7847890

9. Nanda, S. J., Behera, S. K., Panda, G. (2009). Development of a nonlinear model of unijunction transistor using artificial immune system. 2009 World Congress on Nature \& Biologically Inspired Computing (NaBIC). doi: http://doi.org/10.1109/ nabic.2009.5393485

10. Abd El-Basit, W., Awad, Z. I. M., Kamh, S. A., Soliman, F. A. S. (2015). Solar Powered Engine Based on Unijunction Transistors. IOSR Journal of Electrical and Electronics Engineering (IOSR-JEEE), 10 (5 Ver. II), 76-85.

11. Ginoux, J.-M., Meucci, R., Euzzor, S., di Garbo, A. (2018) Torus Breakdown in a Uni Junction Memristor. International Journal of Bifurcation and Chaos, 28 (10), 1850128. doi: http:// doi.org/10.1142/s0218127418501286
12. Di Garbo, A., Euzzor, S., Ginoux, J.-M., Arecchi, F. T., Meucci, R. (2019). Delayed dynamics in an electronic relaxation oscillator. Physical Review E, 100 (3). doi: http://doi.org/10.1103/physreve.100.032224

13. Theraja, B. L., Theraja, A. K. (2005). A Textbook of Electrical Technology, Vol. IV.

14. Jasmine Christina, J., Karthikeyan, V. (2017). Design of Low Power Oscillator for Medical Ultrasonic Sensors with CMUT Implementation. Asian Journal of Applied Science and Techno$\log y, 1$ (1), 68-72.

15. Abd El-Basit, W., El-Ghanam, S. M., Abdel-Maksood, A. M., Kamh, S. A. E.-T., Soliman, F. A. E.-M. S. (2016). Computer Modeling, Characterization, and Applications of Gallium Arsenide Gunn Diodes in Radiation Environments. Nuclear Engineering and Technology, 48 (5), 1219-1229. doi: http:// doi.org/10.1016/j.net.2016.04.009

16. Larin, F. (1968). Radiation Effects in Semiconductor Devices. Wiley: New York.

17. Soliman, F. A. S., Ashry, H. A. (1994). Operation of Some Semiconductors under the Influence of Gamma-radiation. Chinese Journal of Nuclear Science and Engineering, 31 (1), 19-30.

18. Kamh, S. A., Soliman, F. A. S. (2006). Environmental conditions effect on characteristics of some unijunction and bijunction semiconductor devices. Nuclear Instruments and Methods in Physics Research Section A: Accelerators, Spectrometers, Detectors and Associated Equipment, 564 (1), 463-470. doi: http:// doi.org/10.1016/j.nima.2006.03.048

19. Home, W. E. (1970). Literature Search and Radiation Study on Electronic Parts. Final report. Kindle Edition by National Aeronautics and Space Administration NASA. Available at: https:// ntrs.nasa.gov/archive/nasa/casi.ntrs.nasa.gov/19700022435.pdf

20. Bao, J.-L., Zhao, H.-F., Du, L., He, L. Base Resistance in $\mathrm{Si}$ Unijunction Transistor Irradiated by ${ }^{60} \mathrm{Co} \gamma$-Radiation. Actc Physica Sinica, 60 (2), 028501. doi: http://doi.org/10.7498/ aps. 60.028501

Abd El-Basit Wafaa, Assistant Professor, Department of Physics, Ain-Shams University, Cairo, Egypt, ORCID: http://orcid.org/00000002-9059-2980,e-mail: wafaa.abdelbasit@women.asu.edu.eg

Abd El-Maksood Ashraf Mosleh, PhD, Lecturer, Department of Electronics Engineering, Nuclear Materials Authority, Cairo, Egypt, ORCID: http://orcid.org/0000-0001-8502-8042 